\title{
OPEN Effect of berry-based supplements and foods on cognitive function: a systematic review
}

\begin{abstract}
Negar Bonyadi ${ }^{1}$, Neda Dolatkhah ${ }^{2 \bowtie}$, Yaghoub Salekzamani ${ }^{2}$ \& Maryam Hashemian ${ }^{3}$
In the current decade, a growing body of evidence has proposed the correlation between diet and cognitive function or dementia in the ageing population. This study was designed to appraise discoveries from the randomized controlled trials to confirm the effects of berry-based supplements or foods on cognitive function in older adults. PubMed/MEDLINE, Cochrane Central Register of Controlled Trials, Web of Science, Scopus, EMBASE, Google Scholar, and ProQuest as well as SID, Magiran, and Iranmedex electronic databases were explored for human interventional studies up to March 2021. In total, eleven articles were identified using frozen blueberry $(n=4$ studies), blueberry concentrate $(n=2)$, beverage $(n=3)$, capsule $(n=1)$, extract and powder $(n=1)$. These studies had been performed among older people with no recognized cognitive impairment or mild cognitive impairment $(\mathrm{MCl})$. The primary outcomes included global cognitive function, psychomotor function, learning and memory, working memory capacity, executive functions, and brain perfusion/activity. To our knowledge, this is the first systematic review of available clinical trials on the effects of berry-based supplements and foods on cognitive performances as well as brain perfusion parameters among the elderly with normal cognition or $\mathrm{MCl}$. Existing evidence concludes that berry-based supplements and foods have beneficial effects on resting brain perfusion, cognitive function, memory performance, executive functioning, processing speed, and attention indices.
\end{abstract}

Along with the aging of the population worldwide, cognition-related diseases are progressively rising ${ }^{1}$. These disorders, such as mild cognitive impairment (MCI), dementia, and Alzheimer's disease (AD) significantly increase the burden of social and economic health for most communities ${ }^{2}$. The total numeral of individuals with dementia is almost 35.6 million as declared by world health organization (WHO), and by 2050 the total predictable prevalence of AD is anticipated to be 135.5 million $^{3,4}$.

Numerous studies have shown a relationship between lifestyle influences and cognitive function in older adults $^{5-7}$. A considerable amount of data has specified that nutrition is related to age-associated disorders and longevity ${ }^{8,9}$. It has been shown that certain healthy dietary patterns, foods, and micronutrients, such as Mediterranean $\operatorname{diet}^{10}$, vitamin $\mathrm{C}^{11}$, vitamin $\mathrm{E}^{12}$, omega-3 fatty acids ${ }^{13}$, polyphenols, including flavonoids ${ }^{14}$, fruits and vegetables ${ }^{15}$, tea ${ }^{16}$, coffee ${ }^{17}$ and milk ${ }^{18}$, have protective effects on cognitive disorders and dementia.

An initial causal factor to both normal and pathological alterations in brain operation is the agglomeration of oxidative impairment along with diminished endogenous antioxidant barricades ${ }^{19,20}$. The brain is principally exposed to oxidative injury because of its high amount of oxygen intake ${ }^{21}$. Vegetal foods, comprising vegetables, fruits, as well as their juices, are the main human origins of exogenous anti-inflammatory and antioxidant compounds. Additionally, flavonoids are very effective in preserving neurons, improving remaining neuronal operation, increasing neuronal restoration, and inducing neurogenesis ${ }^{22,23}$. Particularly, flavonoids may raise the quantity of between-neuron connections as well as its strength, through their interactions with critical neuronal intracellular signaling paths, which are crucial in adjusting the survivance, differentiation, and longterm potentiation of neurons, and also memor ${ }^{24}$. Though the exact site of flavonoids interaction with signaling paths is uncertain, it seems that they exert their action by connecting to adenosine triphosphate (ATP) locations on receptors and enzymes, modifying the kinase activities directly, affecting the acting of vital phosphatases, functioning in antagonism to kinases, stabilizing $\mathrm{Ca}^{2+}$ homeostasis in neurons, and affecting signaling pathways lying downstream of kinases ${ }^{25,26}$.

\footnotetext{
${ }^{1}$ Student Research Committee, Tabriz University of Medical Sciences, Tabriz, Iran. ${ }^{2}$ Physical Medicine and Rehabilitation Research Center, Aging Research Institute, Tabriz University of Medical Sciences, Tabriz, Iran. ${ }^{3}$ Department of Biology, School of Arts and Sciences, Utica College, Utica, USA. ${ }^{凶}$ email: neda_dolatkhah@ yahoo.com
} 


\begin{tabular}{|l|l|}
\hline Parameter & Description \\
\hline Population & Adult or old subjects with healthy cognition or mild cognitive impairment \\
\hline Intervention & Whole Berries/Berry-based Food/Berry Extract/Berry Supplement \\
\hline Comparator & Any comparator \\
\hline Outcomes & $\begin{array}{l}\text { Outcomes regarding at least one of the following indices: episodic memory, long-term memory, short-term memory, work- } \\
\text { ing memory, executive function, psychomotor reaction time, attention }\end{array}$ \\
\hline Study design & Randomized controlled clinical trial with a crossover or parallel design \\
\hline
\end{tabular}

Table 1. PICOS criteria for inclusion and exclusion of studies.

Preliminary animal studies have established that antioxidant-rich dietary patterns could postpone and even reverse age-associated cognitive decline in laboratory animals ${ }^{27}$. Polyphenols are among the most plentiful antioxidants in the human diet $^{28}$. Recent efforts have proven that berry supplementation (i.e. blueberries and strawberries) can encourage dramatic alterations in the brains of animals besides their currently well-known antioxidant properties ${ }^{29,30}$. The chemical composition of berries is changeable based on the developing locality and ecological environments, plant nourishment, maturity phase, and harvest time, as well as later storage conditions ${ }^{31}$. Main types of polyphenolic flavonoids in berries consist of flavan-3-ols, flavanols, and anthocyanidins ${ }^{32}$. Along with anthocyanins being the principal flavonoids in berries, flavan-3-ols and flavonols are also present in lesser amounts $^{33}$. The health benefits of anthocyanins have been broadly defined, particularly in the prevention of oxidative stress related disorders, such as neurodegenerative diseases ${ }^{34}$. Additionally, current evidence proposes that health-promoting properties of anthocyanins may also be correlated to modification of gut microbiota ${ }^{35}$.

There is some evidence suggesting that polyphenols from berries may be presenting a multiplicity of impacts on the aging brain comprising antioxidant properties, vascular consequences, gluco-regulation, neuro-synthesis, and gut microbiota modifications ${ }^{25,36-41}$. Berry fruits and their chemical ingredients facilitate signaling pathways including those involved in cell longevity as well as increasing neuroplasticity, neurotransmission, and neuronal calcium homeostasis, all of which reduce the age-related decline in behavior ${ }^{40}$. Berry supplementation of animals with damaged hippocampus not only improved the number of neurons surviving but also decreased the amount of stimulated microglia and reduced the pro-inflammatory cytokine expressions ${ }^{42}$. Additionally, berry supplementation was correlated with an improved capability of neurons to preserve learning-related physical and practical adjustments in the hippocampus. Berry supplementation was revealed to persuade stimulation of a multifunctional transcription factor most especially raised throughout the consolidation of short-term to long-term memory in the hippocampus. This activation was related to an improved expression of the growth factor brain-derived neurotrophic factor (BDNF), which has been widely involved in the preservation of neuronal function through aging. Furthermore, dietary blueberry supplementation was revealed to modify the in vivo expression and activity of stress and survival-correlated signaling molecules ${ }^{43}$. Normally consumed berries such as blueberries and strawberries also show anti-glycative activity. ${ }^{44} \mathrm{~A}$ two-percent blueberry diet intake for four months improved appreciation of novelty in old rats ${ }^{45}$. Additionally, a 2-percent blueberry diet improved motor function in old animals and led to enhanced balance plus coordination ${ }^{46}$. However, the available indications for strawberry' improvement of motor performance in old mice and rats are less obvious ${ }^{46-49}$.

Many recent clinical studies have explored the antioxidant and cognition-sparing properties of berries in humans ${ }^{37,50-52}$. Numerous clinical trials have been conducted on the effects of berry-based foods or supplements on cognitive function and memory. Elderly with MCI who consumed blueberry juice $(6-9 \mathrm{~mL} / \mathrm{kg} /$ day) for three months exhibited improved late recall for word lists in the California Verbal Learning Test (CVLT), as compared to the baseline, a tendency to improved functioning, compared to placebo controls and better act in the Verbal Paired Acquaintances Learning Test relative to both baseline and placebo controls ${ }^{53}$.

Due to the lack of systematic review in this regard, the present review study was planned to review recent indications for the beneficial properties of berry-based supplements and foods on cognitive components in the elderly and middle-aged healthy subjects or subjects with MCI.

\section{Methods}

The main objective of the current systematic review was to evaluate the efficacy of whole berries or a berry-based products (e.g. smoothie, juice) or berry extract/capsule consumption in adult or old subjects with healthy cognitions or MCI. The present study has been prearranged according to the guidelines and checklist in Cochrane Handbook for Systematic Reviews of Interventions (version 5.1.0) (54 $^{4}$ and "Preferred Reporting Items for Systematic Reviews and Meta-Analyses (PRISMA)" statement ${ }^{55,56}$. The investigation question was structured based on the PICOS (participants, interventions, comparators, outcomes, and study design) criteria (Table 1) as follows: Do berry-based dietary supplements or foods affect at least one recognized cognition related outcome in adult or old subjects with healthy cognitions or MCI ${ }^{57}$.

Literature search. The online databases (MEDLINE, Web of Science, Cochrane Library, Scopus, EMBASE, Google Scholar, Clininaltrial.gov, Science direct, and ProQuest in addition to SID, Magiran, Irandoc, and Iranmedex for Persian language literature) were employed for search across the titles, abstracts, and keywords of all articles for eligibility up to March 2021. Duplicate studies were excluded. Further, a manual search of the references of the reviewed studies was applied as additional resources to identify other suitable articles that were missed by the electronic search. 
A web-based systematic literature exploration was executed by means of the subsequent MeSH terms: ("randomized controlled trial" OR "RCT" OR "controlled trial" OR "intervention trial" OR "intervention study") AND (Chokeberry OR "Chokeberry extract” OR blackberry OR "blackberry extract" OR raspberry OR "raspberry extract" OR blueberry OR "blueberry extract" OR strawberry OR "strawberry extract" OR cranberry OR "cranberry extract" OR cranberry OR "cranberry extract") AND ("memory"(all Fields) OR "cognitive"(all Fields) OR "cognition"(all Fields) OR "forgetfulness"(all Fields) AND ("older"(all Fields) OR "adult”(All Fields) OR "elderly"(all Fields) OR "older"(all Fields) NOT (animal OR mouse OR mice OR rat OR pig OR cell OR in vitro OR systematic review)). After the primary search, titles and abstracts were sent out from EndNote X8 into Microsoft Excel to be screened. All saved articles were evaluated independently by two investigators (NB and ND) regarding titles and abstracts. Any differences were considered and resolved through consensus or by a third independent investigator (YS).

Inclusion and exclusion criteria. Table 1 reports the PICOS (Participant, Intervention, Comparators, Outcomes, and Study design) criteria accepted in this systematic review of clinical trials. The articles were limited to those published in English or Persian. Inclusion criteria were interventional studies implemented on humans examining the effects of whole berries or a berry-based products (e.g. smoothie, juice) or a berry extract/capsule supplementation on the defined cognitive-related outcomes. The outcomes included global cognitive function, psychomotor function, learning and memory, working memory capacity, executive functions, and brain perfusion/activity in adults and elderlies appraised to have healthy cognitions or MCI. Studies were also excluded if the participants had dementia; if the outcome of the study was not cognitive-related or if the study design was review article, semi- empirical study without a control arm, animal study, trial protocol, letter to the editor, case report, case series, observational study (cross-sectional, case-control and cohort), and unpublished trials.

Data collection. The information of studies regarding the year of publication, participant characteristics, demographic details, location, study design/methodology, interventions (protocol and duration), sample size, dropout and primary outcomes were extracted for each included study by the investigators (NB, ND and YS).

Quality assessment. To assess the risk of systematic errors in the involved trials fulfilling the eligibility criteria, two authors (ND and $\mathrm{MH}$ ) independently estimated the potential risk of bias based on the Cochrane Collaboration's tool for evaluating the risk of bias ${ }^{58}$. Briefly, the trial quality principles consisted of evaluation of: "randomization sequence generation, outcome assessment, blinding of subjects, personal and allocation concealment, imperfect outcome data, and discerning outcome reporting, as well as other sources of bias". Any discrepancies were debated and resolved by agreement or by a third independent reviewer (YS) if required. All trials were determined for each series of bias separately, and the trials were considered to have a score of bias as "low risk," "high risk," or "unclear risk" if the information was insufficient.

The outcome measures. The review's primary outcomes included episodic memory, long-term, and short-term memory, working memory, executive function, psychomotor reaction time, and attention.

\section{Results}

Study selection process. A flowchart explaining the study selection steps is presented in Fig. 1. A total of 259 studies were recognized from the primary database searches, and after eliminating the duplicates, 225 studies remained. Of these, 208 were disqualified after screening the title and abstracts of studies. The remaining 17 studies were evaluated in full text whereby 11 studies fulfilled the eligibility criteria (Fig. 1). The results of quality assessment of included studies is presented as Fig. 2.

Study characteristics. Of the included studies, seven were randomized parallel group $(\mathrm{n}=7)$, two were crossover, and one was a pilot study $(\mathrm{n}=1)($ Table 2$)$.

Cognitive function. Global cognitive performance. Dodd et al. ${ }^{59}$ in a cross-over RCT among 18 healthy older volunteers aged 60-75 years with MMSE score $\leq 25$ reported significantly worse global cognitive performance subsequent the control drink between 2 and $5 \mathrm{~h}$ post-consumption, while there was no significant difference in global cognitive performance between 2 versus $5 \mathrm{~h}$ post-consumption of the blueberry beverage. This means that blueberries could be valuable in terms of preserving cognitive function in older adults. This research had a small sample size and the absence of statistical power may partially describe the non-significant findings in such a relatively heterogeneous sample.

Psychomotor function. Psychomotor performances comprise the combination of accurate motor reactions, attention, and problem-solving capabilities ${ }^{60}$.

Krikorian et al. ${ }^{61}$ detected a trend demonstrating better psychomotor processing speed (shorter time on task) in freeze-dried blueberry powder-treated older adults with MCI in comparison with placebo powder-treated subjects on the Trail- Making Test, part A after 16 weeks of supplementation. One of the main limitations of current trial was the relatively small sample size. Although the findings have been inspiring, there is an obvious necessity for larger trials.

Schrager et al. ${ }^{62}$ studied whether six weeks of daily intake of two cups of frozen blueberry would affect older adults $(n=20)$. They found no significant variations in either the blueberry or placebo groups in simple reaction time with supplementation. Additionally blueberry intake resulted in no significant changes in gait speed under 


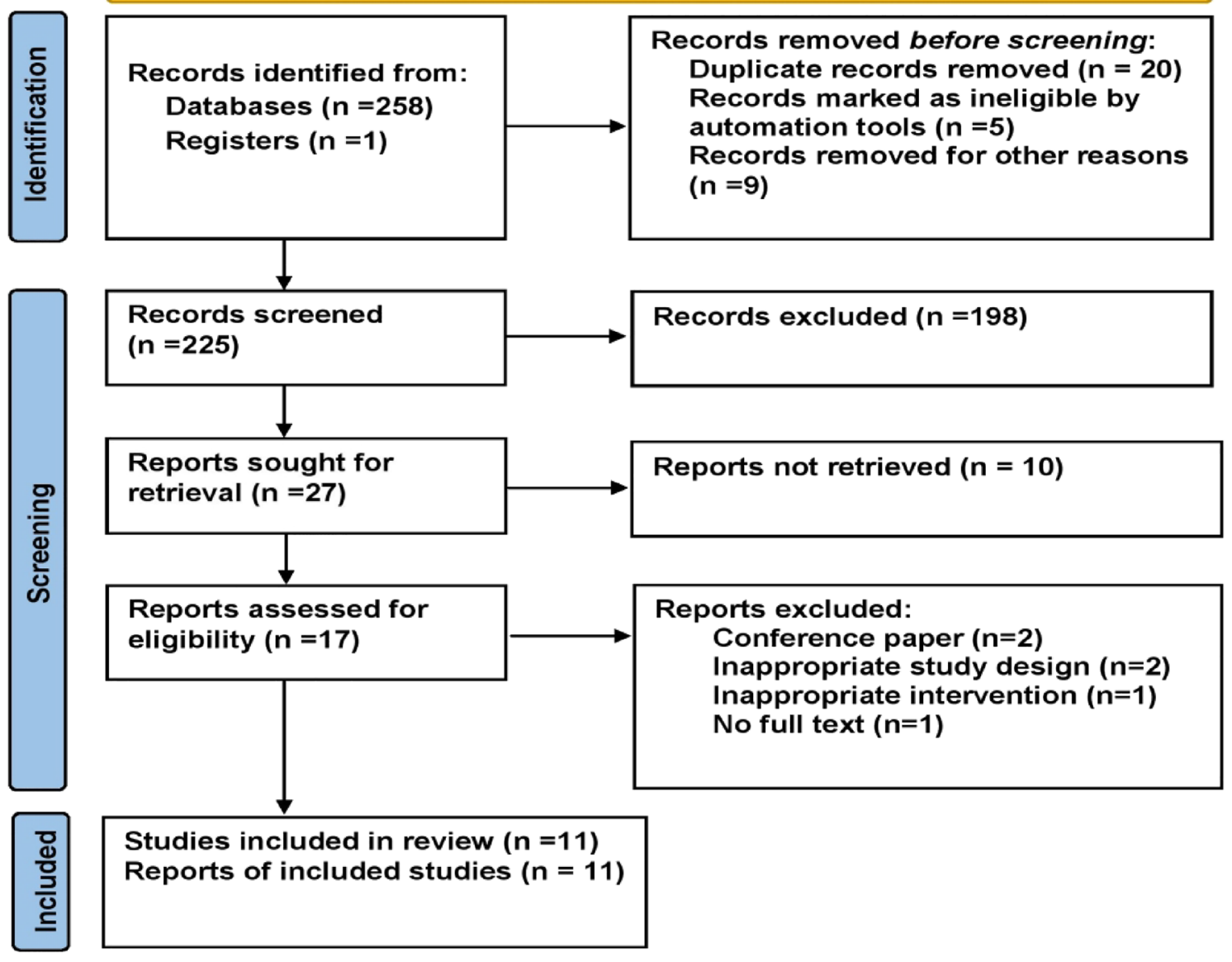

Figure 1. PRISMA flow chart summary of the systematic review search process.

the single-task condition. Substantial within-group improvement in single-task step errors was established in the blueberry group. After adjusting for gait speed, the trend toward a significant improvement in the blueberry group diminished compared to the placebo group in the single-task condition. This study had a small and heterogeneous sample which probably resulted in non-significant results.

Learning and memory. In the RCT by Bensalem et al. ${ }^{63} 6$-month supplementation with polyphenol-rich extract from grape and blueberry (PEGB) led to the higher total number of accurate words at the immediate recall in comparison with the placebo among 60-70-year-old healthy subjects with a BMI between 20-30 and $26<$ MMSE score $\leq 29$. Additionally, performance at the verbal episodic and recognition memory-free recall (VRMFR) was revealed to be affected by PEGB. Furthermore, PEGB led to lower paired associates learning total errors adjusted (PALTEA) among healthy elderly participants in comparison with the baseline scores. A significant interaction was observed between the PEGB effect and the baseline PALTEA score. However, no significant difference was seen between PEGB and placebo groups at the end of the intervention across the entire trial population. PEGB supplementation was established to be considerably more efficient in enhancing cognitive performances among subjects with $\geq 57$ errors in the baseline PALTEA (subjects with the highest cognitive impairments). A high variability in baseline PAL score was observed in this study. Furthermore, the people in the control group had a lower PALTEA score at the second appointment than at baseline, probably because of a test-retest effect. This effect would reduce the likelihood to observe a substantial between-group difference, so the PEGB effects presented in this study may have been underestimated.

In the study of Dodd et al. ${ }^{59}$ intake of a single dose of blueberry beverage resulted in better performance in the immediate word recognition task compared to the control intervention. In spite of no substantial intervention by time interaction, significantly more words were recognized $2 \mathrm{~h}$ after the blueberry consumption compared to the control drink. However, intake of a single dose of blueberry beverage had no beneficiary effect on delayed word recognition task compared to the control intervention. The small sample size may describe the non-significant results of this study.

Bowtell et al. ${ }^{64}$ showed that 12 weeks of daily intake of blueberry concentrate supplementation improved international shopping list task with no significant difference compared to the placebo among 26 healthy older adults. The primary limitation of this study was the small sample size.

Krikorian et al..$^{53}$ examined the efficacy of daily drinking of wild blueberry extract for 12 weeks in a sample of nine older adults with early memory changes. Blueberry juice significantly enhanced the word list recall and 


\begin{tabular}{|c|c|c|c|c|c|c|c|}
\hline Author (year) & Location & Inclusion criteria & $\begin{array}{l}\text { Sample size and } \\
\text { treatment (dosage) }\end{array}$ & $\begin{array}{l}\text { Sample size at the } \\
\text { end of treatment }\end{array}$ & $\begin{array}{l}\text { Design and study } \\
\text { duration }\end{array}$ & Main outcomes & Main results \\
\hline Krikorian et al. ${ }^{53}$ & USA & $\begin{array}{l}\text { Age }>65 \text { years, with } \\
\text { age-related mild, } \\
\text { acquired memory } \\
\text { decline correspond- } \\
\text { ing to MCI }\end{array}$ & $\begin{array}{l}\text { Wild BB juice } \\
6-9 \mathrm{~mL} / \mathrm{kg} / \mathrm{day} \\
(444 \mathrm{~mL} / \mathrm{day} \text { in } \\
\text { individuals weighing } \\
54-64 \mathrm{~kg}, 532 \mathrm{~mL} / \\
\text { day in individuals } \\
\text { weighing } 65-76 \mathrm{~kg} \text {, } \\
\text { and } 621 \mathrm{~mL} / \text { day in } \\
\text { individuals weighing } \\
77-91 \mathrm{~kg})(\mathrm{n}=9)\end{array}$ & - & $\begin{array}{l}\text { Pilot, single-blind?, } \\
\text { one-arm trial, } \\
12 \text { weeks }\end{array}$ & $\begin{array}{l}\text { Verbal learning, } \\
\text { including V-PAL and } \\
\text { CVLT }\end{array}$ & $\begin{array}{l}\text { Improved V-PAL } \\
(p=0.009) \text {, and } \\
\text { CVLT }(p=0.04) \text { fol- } \\
\text { lowing supplemen- } \\
\text { tation }\end{array}$ \\
\hline Schrager et al. ${ }^{62}$ & USA & $\begin{array}{l}\text { Healthy older adults } \\
\text { and age }>60 \text { years old }\end{array}$ & $\begin{array}{l}\text { 1. Flash-frozen BB (2 } \\
\text { cups/day) }(n=13) \\
\text { 2. Placebo/car- } \\
\text { rotjuice drink }(n=7)\end{array}$ & $\begin{array}{l}\text { 1. Flash-frozen BB }(2 \\
\text { cups/day) }(\mathrm{n}=13) \\
\text { 2. Placebo/car- } \\
\text { rotjuice drink }(\mathrm{n}=7)\end{array}$ & $\begin{array}{l}\text { Parallel group, open- } \\
\text { label RCT, } 6 \text { weeks }\end{array}$ & $\begin{array}{l}\text { Psychomotor/adap- } \\
\text { tive gait function and } \\
\text { executive function } \\
\text { (dual-task portion of } \\
\text { the adaptive gait test) }\end{array}$ & $\begin{array}{l}\text { A trend toward a } \\
\text { significant improve- } \\
\text { ment in the BB } \\
\text { group relative to the } \\
\text { placebo }(p=0.065) \\
\text { and significant } \\
\text { relationship between } \\
\text { BB supplementation } \\
\text { group and step error } \\
\text { number under the } \\
\text { dual-task condition } \mathrm{t} \\
(p=0.048)\end{array}$ \\
\hline Nilsson et al. ${ }^{70}$ & Sweden & $\begin{array}{l}\text { Healthy non-smoker } \\
\text { volunteers, age: } \\
50-70 \text { years old, and } \\
\text { BMI } \leq 28 \mathrm{~kg} / \mathrm{m}^{2}\end{array}$ & $\begin{array}{l}\text { 1. Berry beverage } \\
(150 \mathrm{~g} \text { blueberries, } \\
50 \mathrm{~g} \text { blackcurrant, } \\
50 \mathrm{~g} \text { elderberry, } 50 \mathrm{~g} \\
\text { lingonberries, } 50 \mathrm{~g} \\
\text { strawberry, and } \\
100 \mathrm{~g} \text { tomatoes/day) } \\
(\mathrm{n}=23) \\
2 . \text { control } \\
\text { beverage }(\mathrm{n}=23)\end{array}$ & $\begin{array}{l}\text { 1. Berry beverage } \\
(150 \mathrm{~g} \text { blueberries, } \\
50 \mathrm{~g} \text { blackcurrant, } \\
50 \mathrm{~g} \text { elderberry, } 50 \mathrm{~g} \\
\text { lingonberries, } 50 \mathrm{~g} \\
\text { strawberry, and } \\
100 \mathrm{~g} \text { tomatoes/day) } \\
(\mathrm{n}=20) \\
2 . \text { control } \\
\text { beverage }(\mathrm{n}=20)\end{array}$ & $\begin{array}{l}\text { Cross-over open- } \\
\text { label RCT, } 5 \text { weeks }\end{array}$ & $\begin{array}{l}\text { Verbal WM-test, } \\
\text { SA test }\end{array}$ & $\begin{array}{l}\text { Significantly better } \\
\text { performance in WM } \\
\text { test after the berry } \\
\text { beverage compared } \\
\text { to following the } \\
\text { control beverage } \\
(p=0.022)\end{array}$ \\
\hline Bowtell et al. ${ }^{64}$ & England & $\begin{array}{l}\text { Age }>65 \text { years, } \\
\text { ACEIII score of } \\
88 / 100 \text { and higher, } \\
\text { and consuming less } \\
\text { than five portions of } \\
\text { fruit per day }\end{array}$ & $\begin{array}{l}\text { 1. Wild BB sup- } \\
\text { plementation } \\
\text { ( } 30 \mathrm{mLI} \text { concentrate } \\
\text { providing } 387 \mathrm{mg} \\
\text { anthocyanidins }) \\
(\mathrm{n}=12) \\
\text { 2. Isoenergetic } \\
\text { Placebo }(\mathrm{n}=14)\end{array}$ & $\begin{array}{l}\text { 1. Wild BB sup- } \\
\text { plementation } \\
\text { (30 mLlconcentrate } \\
\text { providing } 387 \mathrm{mg} \\
\text { anthocyanidins }) \\
(\mathrm{n}=12) \\
\text { 2. Isoenergetic } \\
\text { Placebo }(\mathrm{n}=14)\end{array}$ & $\begin{array}{l}\text { Parallel group } \\
\text { double-blind RCT, } \\
12 \text { weeks }\end{array}$ & $\begin{array}{l}\text { Brain perfusion, } \\
\text { Task-related activa- } \\
\text { tion, Cognitive } \\
\text { function (psychomo- } \\
\text { tor function, visual } \\
\text { processing, executive } \\
\text { function, verbal and } \\
\text { spatial memory, and } \\
\text { WM) }\end{array}$ & $\begin{array}{l}\text { Significantly better } \\
\text { WM (2-back test) } \\
(p=0.05) \text {, significant } \\
\text { increases in brain } \\
\text { activity within } \\
\text { Brodmann areas } \\
4 / 6 / 10 / 21 / 40 / 44 / 45, \\
\text { precuneus, anterior } \\
\text { cingulate, and } \\
\text { insula/thalamus } \\
\text { (p<0.001), and } \\
\text { significant improve- } \\
\text { ments in grey matter } \\
\text { perfusion in the } \\
\text { parietal }(p=0.013) \\
\text { and occipital } \\
(p=0.031) \text { lobes after } \\
\text { blueberry vs. placebo } \\
\text { supplementation }\end{array}$ \\
\hline Whyte et al. ${ }^{65}$ & England & $\begin{array}{l}\text { Independently living } \\
\text { healthy volunteers, } \\
\text { age: } 65-80 \text { years old, } \\
\text { all ethnicity, and sub- } \\
\text { jective self-reported } \\
\text { memory complaints }\end{array}$ & $\begin{array}{l}\text { 1.WBP }(500 \mathrm{mg} / \\
\text { day) }(\mathrm{n}=30) \\
\text { 2. WBP }(1000 \mathrm{mg} / \\
\text { day) }(\mathrm{n}=31) \\
\text { 3.WBE }(111 \mathrm{mg} / \\
\text { day) }(\mathrm{n}=31) \\
\text { 4.Placebo }(\mathrm{n}=30)\end{array}$ & $\begin{array}{l}\text { 1.WBP }(500 \mathrm{mg} / \\
\text { day) }(\mathrm{n}=28) \\
\text { 2. WBP }(1000 \mathrm{mg} / \\
\text { day) }(\mathrm{n}=29) \\
\text { 3.WBE }(111 \mathrm{mg} / \\
\text { day) }(\mathrm{n}=28) \\
\text { 4.Placebo }(\mathrm{n}=27)\end{array}$ & $\begin{array}{l}\text { Parallel group } \\
\text { double-blind RCT, } \\
6 \text { months }\end{array}$ & \begin{tabular}{|l|} 
Verbal (RAVLT), \\
visual (Corsi Block) \\
and short term spa- \\
tial episodic memory, \\
WM, and executive \\
function including \\
selective attention
\end{tabular} & $\begin{array}{l}\text { Significantly better } \\
\text { performance in } \\
\text { verbal episodic } \\
\text { memory }(p=0.038) \text {, } \\
\text { and a trend towards } \\
\text { better performance } \\
\text { in visual episodic } \\
\text { memory following } \\
\text { WBE111 interven- } \\
\text { tion in comparison } \\
\text { to placebo }(p=0.069)\end{array}$ \\
\hline Miller et al. ${ }^{66}$ & USA & $\begin{array}{l}\text { Age: } 60-75 \text { years, } \\
\text { BMI: } 18.5-29.9, \\
\text { adequate visual acu- } \\
\text { ity, and } \\
>12 \text { months post- } \\
\text { menopausal }\end{array}$ & $\begin{array}{l}\text { 1.freeze-dried BB } \\
(24 \mathrm{~g} / \text { day })(\mathrm{n}=20) \\
\text { 2. Placebo }(\mathrm{n}=20)\end{array}$ & $\begin{array}{l}\text { 1.freeze-dried BB } \\
\text { (24 g/day) }(\mathrm{n}=19) \\
\text { 2. Placebo }(\mathrm{n}=19)\end{array}$ & $\begin{array}{l}\text { Parallel group } \\
\text { double-blind RCT, } \\
90 \text { Days }\end{array}$ & $\begin{array}{l}\text { Executive func- } \\
\text { tion (TST, TMT), } \\
\text { long-term memory } \\
\text { (CVLT-II), short- } \\
\text { term memory (DS } \\
\text { task), spatial cogni- } \\
\text { tion (VMWM), and } \\
\text { attention (ANT) }\end{array}$ & $\begin{array}{l}\text { Significantly } \\
\text { fewer repetition } \\
\text { errors in the CVLT } \\
(p=0.031) \text { and } \\
\text { reduced switch cost } \\
\text { on a } \\
\text { TST ( } p=0.033 \text { ) } \\
\text { across study visits } \\
\text { following freeze- } \\
\text { dried BB interven- } \\
\text { tion in comparison } \\
\text { with placebo }\end{array}$ \\
\hline
\end{tabular}




\begin{tabular}{|c|c|c|c|c|c|c|c|}
\hline Author (year) & Location & Inclusion criteria & $\begin{array}{l}\text { Sample size and } \\
\text { treatment (dosage) }\end{array}$ & $\begin{array}{l}\text { Sample size at the } \\
\text { end of treatment }\end{array}$ & $\begin{array}{l}\text { Design and study } \\
\text { duration }\end{array}$ & Main outcomes & Main results \\
\hline Boespflug et al. ${ }^{92}$ & USA & $\begin{array}{l}\text { Age }>65 \text { years, } \\
\text { with mild cognitive } \\
\text { impairment consist- } \\
\text { ent with MCI }\end{array}$ & $\begin{array}{l}\text { 1. Whole freeze- } \\
\text { dried BB powder } \\
\left(12.5 \mathrm{~g} \text { per packet }{ }^{\star} 2 /\right. \\
\text { day })(\mathrm{n}=11) \\
\text { 2. Placebo powder } \\
(\mathrm{n}=10)\end{array}$ & $\begin{array}{l}\text { 1. Whole freeze- } \\
\text { dried BB powder } \\
\left(12.5 \text { g per packet }{ }^{\star} 2 /\right. \\
\text { day }(\mathrm{n}=8) \\
\text { 2. Placebo powder } \\
(\mathrm{n}=8)\end{array}$ & $\begin{array}{l}\text { Parallel group } \\
\text { double-blind RCT, } \\
16 \text { weeks }\end{array}$ & $\begin{array}{l}\text { WM performance, } \\
\text { and functional MRI }\end{array}$ & $\begin{array}{l}\text { Increased BOLD } \\
\text { activation in the left } \\
\text { pre-central gyrus, } \\
\text { left middle frontal } \\
\text { gyrus, and left } \\
\text { inferior parietal lobe } \\
\text { during WM load } \\
\text { conditions ( }<<0.01 \text { ) } \\
\text { with no clear } \\
\text { enhancement of WM } \\
\text { following freeze- } \\
\text { dried BB interven- } \\
\text { tion in comparison } \\
\text { with placebo }\end{array}$ \\
\hline McNamara et al. ${ }^{68}$ & USA & $\begin{array}{l}\text { Age: } 62-80 \text { years } \\
\text { old, and mild, self- } \\
\text { perceived cognitive } \\
\text { decline differenti- } \\
\text { ated from MCI and } \\
\text { prodromal AD }\end{array}$ & $\begin{array}{l}\text { 1. FO }(400 \mathrm{mg} \\
\text { EPA and } 200 \mathrm{mg} \\
\mathrm{DHA} \times 4 / \text { day })+ \\
\text { placebo powder } \\
(\mathrm{n}=21) \\
\text { 2. freeze dried } \mathrm{BB} \\
\text { powder }\left(12.5 \mathrm{~g}^{\star} 2 /\right. \\
\text { day }+ \text { placebo oil } \\
(\mathrm{n}=24) \\
\text { 3. FO }+\mathrm{BB} \\
\text { Powder }(\mathrm{n}=26) \\
\text { 4. Placebo }(\mathrm{n}=23)\end{array}$ & $\begin{array}{l}\text { 1. FO }(400 \mathrm{mg} \\
\text { EPA and } 200 \mathrm{mg} \\
\text { DHA } \times 4 / \text { day })+ \text { pla- } \\
\text { cebo powder }(\mathrm{n}=15) \\
\text { 2. freeze dried BB } \\
\text { powder }\left(12.5 \mathrm{~g}^{\star} 2 /\right. \\
\text { day })+ \text { placebo oil } \\
(\mathrm{n}=16) \\
\text { 3. FO }+\mathrm{BB} \\
\text { Powder }(\mathrm{n}=17) \\
\text { 4.Placebo }(\mathrm{n}=17)\end{array}$ & $\begin{array}{l}\text { Parallel group } \\
\text { double-blind RCT, } \\
24 \text { weeks }\end{array}$ & $\begin{array}{l}\text { Psychomotor speed, } \\
\text { WM, lexical access, } \\
\text { new learning and } \\
\text { long-term memory } \\
\text { (HVLT), and cogni- } \\
\text { tive symptoms in } \\
\text { everyday activities } \\
\text { (DEX) }\end{array}$ & $\begin{array}{l}\text { Improved DEX } \\
\text { following FO supple- } \\
\text { mentation compared } \\
\text { to placebo }(p=0.03) \\
\text { and following BB } \\
\text { supplementation } \\
\text { compared to placebo } \\
(p=0.05) \\
\text { Improved HVLT for } \\
\text { the BB-treated group } \\
\text { compared to placebo } \\
(p=0.04)\end{array}$ \\
\hline Bensalem et al. ${ }^{63}$ & France \& Canada & \begin{tabular}{|l|} 
Age: $60-70$ years \\
old healthy subjects, \\
BMI: $20-30$, \\
$26<$ MMSE score $\leq 29$ \\
and usual dietary \\
intake of red fruits $\leq 2$ \\
servings per week; \\
tea consumption $\leq 1$ \\
cup per day; dark \\
chocolate $(\geq 70 \%$ \\
cocoa) $\leq 140$ g per \\
week and less than 3 \\
servings per week of \\
omega-3 fatty acids \\
rich foods such as \\
fish, algae,...
\end{tabular} & $\begin{array}{l}\text { 1. } 300 \mathrm{mg}^{\star} 2 / \text { day } \\
\text { PEGB capsule } \\
\text { (containing } 258 \mathrm{mg} \\
\text { flavonoids) }(\mathrm{n}=101) \\
\text { 2. placebo }(\mathrm{n}=105)\end{array}$ & $\begin{array}{l}\text { 1. } 300 \mathrm{mg}^{\star} 2 / \text { day } \\
\text { PEGB capsule } \\
\text { (containing } 258 \mathrm{mg} \\
\text { flavonoids) }(\mathrm{n}=92) \\
\text { 2. placebo }(\mathrm{n}=98)\end{array}$ & $\begin{array}{l}\text { Parallel group } \\
\text { double-blind RCT, } \\
6 \text { months }\end{array}$ & $\begin{array}{l}\text { PALTEA, VRM, and } \\
\text { WM }\end{array}$ & $\begin{array}{l}\text { Improved cognitive } \\
\text { performances of par- } \\
\text { ticipants with base- } \\
\text { line PALTEA } \geq 57 \\
\text { errors, improved } \\
\text { PALTEA ( } p=0.037) \text {, } \\
\text { VRMFR }(p=0.014) \\
\text { and the delayed } \\
\text { version of the } \\
\text { VRMR ( } p=0.005) \\
\text { with no significant } \\
\text { enhancement of } \\
\text { WM following } \\
\text { PEGB intervention } \\
\text { in comparison with } \\
\text { placebo }\end{array}$ \\
\hline Dodd et al. ${ }^{59}$ & UK & $\begin{array}{l}\text { Age: } 60-75 \text { years, } \\
\text { MMSE score: } \leq 25, \\
\text { and a depression } \\
\text { index score on BSI } \\
: \geq 11\end{array}$ & $\begin{array}{l}\text { 1.a single dose } \\
\text { flavonoid rich } \mathrm{BB} \\
\text { beverage ( } 508 \mathrm{mg} \\
\text { of antho- and } \\
71 \mathrm{mg} \text { procya- } \\
\text { nidins) equivalent to } \\
\text { approximately } 200 \mathrm{~g} \\
\text { of fresh BB } \\
2 . \text { Placebo } \\
\text { beverage }(\mathrm{n}=18)\end{array}$ & & $\begin{array}{l}\text { Cross-over single- } \\
\text { blind? RCT } \\
2 \text { h versus } 5 \mathrm{~h}\end{array}$ & $\begin{array}{l}\text { Executive function } \\
\text { and memory (Go- } \\
\text { NoGo, Stroop, Digit } \\
\text { Switch, Continuous } \\
\text { Performance Task, } \\
\text { Digit Symbol Substi- } \\
\text { tution Test, Random } \\
\text { Word Generation, } \\
\text { Three-Word Sets } \\
\text { Task, N-back, Letter } \\
\text { memory, Location } \\
\text { Task, immediate and } \\
\text { delayed recall and } \\
\text { recognition) }\end{array}$ & $\begin{array}{l}\text { Significantly worse } \\
\text { performance follow- } \\
\text { ing the control drink } \\
\text { at } 2 \mathrm{~h} \text { compared to } \\
5 \mathrm{~h} \text { post consump- } \\
\text { tion ( } p=0.04) \text {, better } \\
\text { performance in the } \\
\text { immediate word } \\
\text { recognition task fol- } \\
\text { lowing the blueberry } \\
\text { compared with the } \\
\text { control interven- } \\
\text { tion ( } p=0.05) \text {, and } \\
\text { significantly more } \\
\text { word recognition } \\
\text { following the blue- } \\
\text { berry compared to } \\
\text { the control drink at } \\
\text { the } 2 \mathrm{~h}(p=0.02)\end{array}$ \\
\hline Krikorian et al. ${ }^{61}$ & USA & $\begin{array}{l}\text { Age } \geq 68 \text { years, with } \\
\text { MCI confirmed }\end{array}$ & $\begin{array}{l}\text { 1. Whole BB powder } \\
\text { packet contained } \\
12 \text { g powder }{ }^{\star} 2 / \text { day } \\
(\mathrm{n}=24) \\
2 . \text { Placebo powder } \\
10 \text { g powder }{ }^{\star} 2 / \text { day } \\
(\mathrm{n}=23)\end{array}$ & $\begin{array}{l}\text { 1. Whole BB powder } \\
\text { packet contained } \\
12 \text { g powder }{ }^{\star} 2 / \text { day } \\
(\mathrm{n}=16) \\
2 . \text { Placebo powder } \\
10 \text { g powder }{ }^{\star} 2 / \text { day } \\
(\mathrm{n}=21)\end{array}$ & $\begin{array}{l}\text { Parallel double-blind } \\
\text { group RCT, } 16 \text { weeks }\end{array}$ & $\begin{array}{l}\text { Speed of processing, } \\
\text { WM, lexical access } \\
\text { for semantic access, } \\
\text { and verbal and } \\
\text { nonverbal long-term } \\
\text { memory }\end{array}$ & $\begin{array}{l}\text { Improved semantic } \\
\text { access } \mathrm{s}(p=0.01) \\
\text { and better SPAL } \\
(p=0.05) \text { following } \\
\text { the BB powder com- } \\
\text { pared to the placebo } \\
\text { powder }\end{array}$ \\
\hline
\end{tabular}

Table 2. Included randomized controlled trials of berry-based food interventions on cognitive function. AD: Alzheimer's disease 'AN: Attention Network Task, BB:blueberry, BMI: body mass index, BSI: the Brief Symptom Inventory, CVLT: the California Verbal Learning Task, CVLT-II: California Verbal Learning Test, 2nded, DEX: Dysexecutive questionnaire, DS: digit span, FO: fish oil; GDS: Geriatric Depression Scale, HVLT: Hopkins Verbal Learning Test, MCDR: modified Clinical Dementia Rating, MCI: mild cognitive impairment, MMSE: a Mini Mental State Examination, MoCA: Montreal Cognitive Assessment, PAL: Paired Associate Learning Test, PALTEA: PAL total errors adjusted, PEGB :polyphenol-rich extract from grape and blueberry, POMS: Profile of Mood States, RAVLT: Rey's Auditory Verbal Learning task, RCT: randomized double-blind controlled trials, SA: Selective attention, TST: task-switching test, TMT: trail-making test, VMWM: virtual version of the Morris Water Maze, V-PAL: Verbal Paired Associate Learning Test, VRM: verbal episodic and recognition memory, WBE: Wild Blueberry extract, WBP: Wild Blueberry Powder, WM: Working memory. 


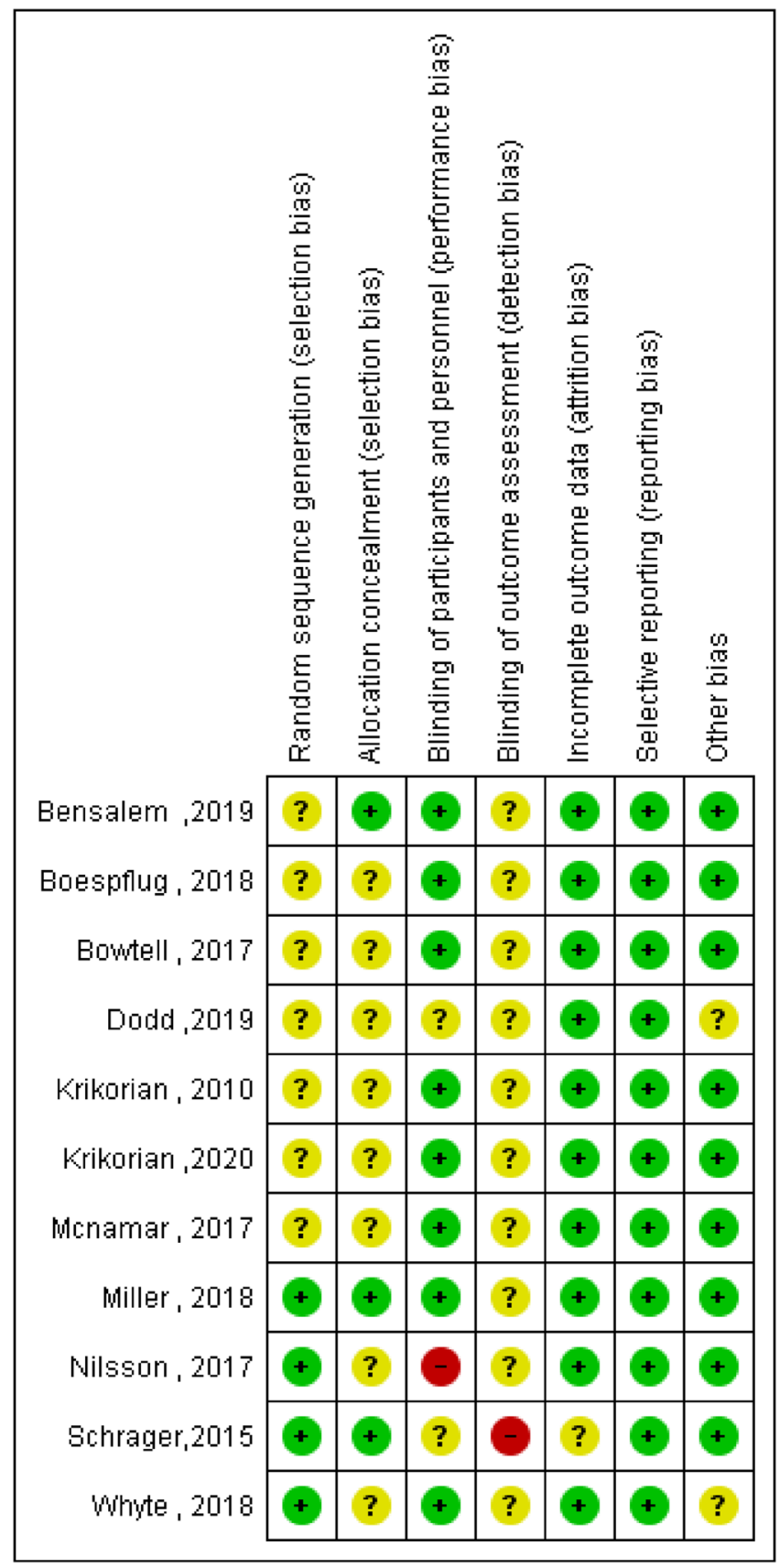

Figure 2. Risk of bias.

paired associate learning. The discoveries of this trial propose that moderate-term supplementation with blueberry can provide a neurocognitive advantage.

The small sample size and lack of a completely matched control product were the primary limitations of this study. However, the relatively greater glycemic content of the grape juice as the placebo beverage may have influenced the cognitive performance.

The word recognition procedure wants the participants to identify previously presented words from the main word list whereas ignoring words from another word list and further phonetically or semantically matched diverse words. It has been known that episodic memory performance would decrease in older people ${ }^{65}$.

In a double-blinded, placebo-controlled RCT by Whyte et al ${ }^{65}$ daily supplementation with purified wild blueberry extract at $100 \mathrm{mg}$ (WBE111) for six months resulted in a significantly improved function in late word recognition in the Reys Auditory Verbal Learning Task (RAVLT) (in contrast with the placebo in 65-80-year-old healthy subjects with self-reported memory complaints). These impacts were not seen for whole wild blueberry powder at $500 \mathrm{mg}$ (WBP500) and $1000 \mathrm{mg}$ (WBP1000). Furthermore, daily WBE111 supplementation caused an improving trend in visuospatial Corsi Block function (the entire number of orders properly recalled) in contrast with the placebo after 3 months of supplementation. 
Miller et al. ${ }^{66}$ showed that 3-month supplementation of 37 men and women (age 60-75 years) with MMSE score $\geq 24$ with freeze-dried blueberry resulted in fewer repetition faults according to CVLT, 2 nd Ed. ${ }^{67}$ after the end of intervention than they did at the baseline.

In the RCT by Krikorian et al. ${ }^{61}$ freeze-dried blueberry fruit powder supplementation resulted in a significantly better nonverbal memory performance (SPAL) compared to the placebo powder in adults aged 68 years and older with MCI. In spite of the obvious improvement in the Hopkins Verbal Learning Test (HVLT) recall favoring the blueberry group, it was not a significant effect. An effect was observed also for semantic access and not for phonological access favoring the blueberry-supplemented group. The relatively low sample size may partially describe the non-significant findings in this sample.

McNamara et al. ${ }^{68}$ examined the effect of 24 -week supplementation with daily fish oil (EPA and DHA) or blueberry or both in elderly men and women aged 62-80 years old with mild, self-perceived cognitive deterioration in a randomized, double- blind, placebo-controlled trial. This was monitored for a further 24 weeks. There was an effect demonstrating enhanced insight in recognition memory on the HVLT for the blueberry-treated group. The memory insight improvement in the blueberry group was not preserved at week 48 (24 weeks following cessation of the supplementation).

Working memory (WM) capacity. WM represents the limited quantity of info that can be kept in mind and used in the practical implementation of cognitive jobs. It has often been correlated to intelligence, data processing, executive function, understanding, problem-solving, and learning ${ }^{69}$.

Nilsson et al..$^{7}$ in a randomized crossover trial showed that supplementation for five weeks among 46 healthy men and women within 50-70 years of age with a berry drink based on a combination of berries (blueberries, blackcurrant, elderberry, lingonberries, strawberry, and tomatoes) enhanced the performance significantly in the WM-test at $30 \mathrm{~min}$ by nearly $5 \%$ in contrast to the control beverage. The test employed in the current study demonstrated an extension of the Radeborg et al. developed methodology ${ }^{71}$. The tests consisted of twelve sets of short indicative sentences that could be either meaningful 'the boy came back from school', or absurd, such as 'the cat struck the concept'. The sentences were read to the participants, and instantly after each sentence the participant had to specify if the sentence was meaningful or not. After each set of sentences, the participants had to repeat the first noun in each of the sentences. An apparent limitation of this study was that it was not probable to blind the products to the participants because of the evident dissimilarities. Furthermore, the test product included a mix of berries which discard possibilities to provide any suggestion concerning individual berries.

Krikorian et al ${ }^{61}$ found no effect for task switching as measured by the Trail-Making Test, part B task following blueberry supplementation in older adults with MCI compared to the placebo. No notable effect of PEGB was observed either by Bensalem et al. ${ }^{63}$ for the WM measured through the Spatial Span (SSP) and the Reverse SSP.

In an RCT by Whyte et al. ${ }^{65}$ daily supplementation with WBP or WBE for six months was not associated with encouraging impacts in older adults. WM was assessed through implementation of two different tasks, involving serial subtractions as designated beforehand by Bell et al. ${ }^{72}$ plus Sternberg memory scanning. Probably, an element of practice effect occurred whereby, participants enhanced their approach on performance in these memory tasks thus decreasing the task sensitivity to the treatment. Additionally, possible deterioration of the capsule active constituents at 6 months and increased tolerance to the WBE are other issues to be considered.

Executive function. Miller et al. ${ }^{66}$ reported that 3-month supplementation of 37 men and women (ages 60-75 years) with MMSE score $\geq 24$ with freeze-dried blueberry resulted in a greater reduction in switch stimuli errors between follow-ups in task-switching test (TST) [a practice visit (visit 1), a baseline visit (visit 2), and 45and 90-day interposition visits (visits 3 and 4, correspondingly)] in comparison with the control. When group differences in physical activity and computer use were measured, the interposition endured. Post hoc analysis showed that, within the blueberry group, performance on the baseline and 90-day intervention visits as well as 45- and 90-day interposition visits were meaningfully different even when the Bonferroni modification was exerted.

In the study by Dodd et al. ${ }^{59}$ a tendency towards lower switch cost was detected following the blueberry usage in comparison with the control beverage at the 2 -h time point; however, the overall intervention by time interaction proved insignificant.

Bowtell et al. ${ }^{64}$ showed that 12 weeks of the daily intake of a blueberry concentrate in healthy older adults improved WM and also the Groton maze learning role in comparison with the placebo. The WM was assessed by means of an n-back task, including 1-back and 2-back memory task blocks. Although the trial did not report significant effects in cognitive function, the percentage change in presentation of the 2-back tests showed a piece of weak evidence for upgrading in the blueberry versus the placebo. Although the findings are encouraging, there is a clear need for larger trials.

Krikorian et al. ${ }^{61}$ observed no effect for perceived cognitive efficiency in daily tasks as measured by the dysexecutive questionnaire (DEX) after 16 weeks of supplementation with blueberry powder in comparison with the placebo.

Daily consumption of two cups of frozen blueberry for 6 weeks resulted in a significant improvement in foot placement and balance control during computerized dual-task testing of gait compared to the placebo in the Schrager et al.'s study ${ }^{62}$.

In the Whyte et al.s study ${ }^{65}$, daily supplementation with WBE or WBP was correlated with no promising impacts on executive function and attention measured by modified attention network task (MANT) as described by Whyte et al. ${ }^{73}$ Meanwhile, there were only two blocks with a stimulus length of $500 \mathrm{~ms}$, with no noise condition applied. 
Brain perfusion/activity. Bowtell et al. ${ }^{64}$ showed that 12 weeks of the daily intake of a blueberry concentrate in healthy older adults resulted in significant improvements in brain activity in response to blueberry supplementation compared to the placebo group within Brodmann zones 4/6/10/21/40/44/45, precuneus, anterior cingulate cortex (ACC), and insula/thalamus, along with significant enhancements in grey matter perfusion in the parietal plus occipital lobes.

Brodmann area 21 [middle temporal gyrus (MTG)] is implicated in sound recognition as well as word and picture semantic processing ${ }^{74}$, language processing ${ }^{75}$, decoding gaze direction, deductive reasoning ${ }^{76}$ plus intelligible speech and processing of verbal mental arithmetic ${ }^{77}$. Brodmann area 40 (supramarginal gyrus) plays a role in visual word recognition, auditory memory processing, and emotion recognition ${ }^{78-80}$. The left-hemisphere Brodmann areas 44 and 45 are associated with language processing (including production and comprehension) ${ }^{81}$ plus higher cognitive functions including music, calculus, and $\mathrm{WM}^{82}$. The area 44 is involved in conditions of vicarious learning, i.e., attentive consideration of activities of another ${ }^{83}$. ACC plays a substantial role in attention, performance monitoring, error processing, emotional information processing, and motivational behavior ${ }^{84-88}$. Improved ACC activity is related to faster speed in a reaction time task ${ }^{89}$. The insula has various roles in humans from sensory and affective processing to high-level cognition (attention plus salience processing and speech) $)^{90}$. The thalamus is an important node in linkages enduring cognitive performances, identified to deteriorate in usual aging, comprising procedures of memory as well as executive functions and information processing ${ }^{91}$.

Boespflug et al. ${ }^{92}$ in a double-blind, placebo-controlled RCT assessed the effect of daily blueberry supplementation or placebo powder for 16 weeks on blood oxygen level-dependent (BOLD) signal in sixteen 68-year and older adults with MCI. Blueberry-treated patients displayed augmented BOLD activation in the left pre-central gyrus, left middle frontal gyrus (LMFG), and left inferior parietal lobe throughout working memory load situations. A rising number of investigations have studied how motivation interrelates with particular cognitive performances, comprising attention, WM, and other executive functions. It's true for a subset of brain regions, which included the left pre-central gyrus ${ }^{93}$. The LMFG plays a crucial role in the progress of executive attention, literacy, and word production ${ }^{94-96}$. Left inferior parietal lobe is implicated in language comprehension plus production and converges semantic information pathways ${ }^{97,98}$.

\section{Discussion}

To our knowledge, this is the first systematic review of available clinical trials on the effects of berry-based supplements and foods on cognitive performances and brain perfusion parameters in elderlies with normal cognition or MCI. This review of 11 clinical trials provided inconsistent findings across the measured outcomes. Given the heterogeneity of study design, intervention type/dose/duration, outcomes of interest and cognitive tasks used, the described trials have revealed that berry-based supplements and foods may have beneficial effects on global cognitive performance (one study) $)^{59}$, psychomotor function (two studies) ${ }^{61,62}$, learning and memory (nine studies) ${ }^{53,59,61,63-66,68}$, WM capacity (three studies) ${ }^{61,65,70}$,executive function (six studies) ${ }^{59,61,62,64-66}$, and brain perfusion/ activity (two studies) ${ }^{64,92}$. Nine of 11 trials were of poor or modest methodological quality principally because of suspicions about the blinding of outcome assessment, allocation concealment and random allocation to groups. The exact interventions given in the included trials were heterogeneous and in some trials poorly explained.

Fruit juice and powder may be impressive approaches to enhancing the total fruit intake. Regarding the nutritional worth, freeze-dried powder that is free of water maintains concentrated bio-available antioxidants, fiber, and other ingredients ${ }^{99}$. Some studies have proposed that the juicing procedure can result in a poor content of fiber and some bio-actives comprising polyphenols, vitamins, and minerals ${ }^{100,101}$, while another study suggests that processing can improve the carotenoid bioavailability ${ }^{102}$. On the other hand, the microstructure of the extract in contrast to the powders must also be considered. The extract lacks fibers and is water-soluble, while the full spectrum powders are very rich in insoluble fibers to which the polyphenols are bound. It is thus likely to assume that the polyphenols in the powders would not be as bioavailable as in the extract, and may hence be less worthwhile.

Most included studies had small sample sizes which may explain the non-significant findings. In one study, the product involved a mix of berries which made it impossible to give any conclusion regarding individual berries. In studies with longer duration of treatment, possible deterioration of the capsule's active constituents and increased tolerance to the product are other concerns. In most included studies, beneficial properties were established with dosage, which is achievable for daily consumption. Although other studies used higher amounts, this may only be possible for a limited therapeutic period. Nonetheless, establishing the involvement of berry metabolites in the cognition is puzzling, chiefly because berries have a different phytochemical profile, which can be extremely different across varieties.

Berries can be found as various little red, purple, or blue fruits. The frequently consumed berries include the blackberries (Rubus spp.), blueberries (Vaccinium corymbosum), cloudberry (Rubus chamaemorus L.), cranberry (Vaccinium macrocarpon), red raspberries (Rubus idaeus), black raspberries (Rubus occidentalis L.), and strawberries (Fragaria x ananassa). Less frequently consumed berries are black currants (Ribes nigrum), chokeberries (Aronia melanocarpa), lingonberry (Vaccinium vitis-idaea L.) and mulberries (Morus alba L.). Berries are eaten both as fresh fruit in addition to processed food products (for example beverages, extracts, jams, jellies, and freeze-dried). Generally, berries have low calories and high fiber, and contain different natural antioxidants such as vitamins $\mathrm{C}$ and $\mathrm{E}$, plus other nutrients such as folic acid, calcium, selenium, alpha and beta carotene, and lutein. Berries are rich in polyphenols, with significant amounts of flavonoids (anthocyanins, flavonols, and flavanols), condensate tannins (proanthocyanidins), hydrolyzable tannins (ellagitannins and gallotannins), phenolic acids (hydroxybenzoic and hydroxycinnamic acids, chlorogenic acid), stilbenoids, and lignans ${ }^{103,104}$. Anthocyanins are a major group of natural, water-soluble pigments and provide the bright appearance to berries. Around 400 
anthocyanins have been distinguished, which are mostly concentrated in the fruit external layer, particularly berries. Red berries, strawberries, and cherries have also anthocyanins in their body ${ }^{105}$.

When findings of the dietary intermediations systematically reviewed in this study are weighed together, it seems that there are numerous essential mechanisms whereby berries may potentially provide cognitive health properties. The brain is predominantly exposed to neuro-inflammation and oxidative stress. This susceptibility further grows with age $\mathrm{f}^{70}$. Amplified reactive oxygen species (ROS) production or reduced antioxidant protection is described as oxidative stress, which may contribute to the increase in various disorders such as neurodegenerative diseases ${ }^{106}$. Several medicinal properties of berries against oxidative stress-related disorders have been associated with their great content of phenolic antioxidants, particularly anthocyanin and phenolic acids. The antioxidant effects of anthocyanins were evaluated in vitro, comprising 1,1-diphenyl-2-picrylhydrazyl, radical scavenging property, oxygen radical absorbance capability and ferric decreasing antioxidant potential ${ }^{107-109}$. Furthermore, berries have proved to contain an acceptable content of vitamin A, vitamin, $\mathrm{C}$ and vitamin $\mathrm{E}$, which function as antioxidants ${ }^{31}$. On the other hand, it has been discussed that inflammatory cytokines in the circulation can cross the blood-brain barrier (BBB), initiate a neuro-inflammatory condition, disturb neuroendocrine performance as well as neurotransmitter arrangements, finally resulting in cognitive impairment ${ }^{110}$. The transcription factor nuclear factor kappa-light-chain-enhancer of activated B cells (NF- $\kappa \mathrm{B})$ signaling pathway has a critical role in organizing inflammatory reactions ${ }^{111}$. Anthocyanin shuts off the initiation of NF- $\kappa \mathrm{B}$ and activation of nitric oxide synthase-2, cyclooxygenase-2, interleukin-1 $\beta$ (IL-1 $\beta$ ), and tumor necrosis factor- $\alpha$ (TNF- $\alpha$ ) in macrophages ${ }^{112,113}$. Furthermore, anthocyanins are correlated with down-regulation of inflammatory cytokine genes including interleukin- 6 and monocyte chemo-attractant protein- 1 in adipose tissue ${ }^{114}$. Berry polyphenols improve mitochondrial function in intestinal Caco-2/15 cells activated with lipopolysaccharide (LPS), which reduces intestinal inflammation ${ }^{115}$. Metabolites of anthocyanin imitate active agents such as the anti-inflammatory salicylic acid (2-hydroxybenzoic acid) and are associated with useful variations in biomarkers of inflammation in in-vitro models ${ }^{35,116}$. A rodent model also revealed that anthocyanin intervention suppressed the biological activity of cyclooxygenase- $2^{117}$. So anthocyanins decrease the omega- 6 eicosanoids turnover (for example, prostaglandin E2 (PGE2) and leukotriene B4 (LTB4)). Additionally, anthocyanin microbial metabolites, such as 3,4-dihydroxybenzoic acid (protocatechuic acid), 4-hydroxy-3-methoxybenzoic acid (vanillic acid), 4-hydroxy-3,5-dimethoxybenzoic acid (syringic acid), and 3,4,5-trihydroxybenzoic acid (gallic acid), are related with advantageous variations in biomarkers of inflammation in in-vitro models ${ }^{118}$.

Meanwhile, phenolic compounds are shown to be extensively metabolized into simple phenolic metabolites through the action of microorganisms in the colon ${ }^{119}$. These compounds may modify gut microbiota by stimulating favorable bacteria and deterring pathogenic bacteria. In healthy individuals, the gut microbiota modulations largely result in an increase in Bifidobacterium, Lactobacillus and Akkermansia, thus proposing a prebiotic-like property of the berries or their compounds ${ }^{119-122}$. Epidemiological explanations and studies in animal models confirm a common schema that involves the gut microbiota over the microbiome-gut-brain axis in the pathogenesis of neurodegenerative diseases such as dementia ${ }^{123}$.

The current study had some limitations that should be considered in explaining the outcomes of this systematic review. First, despite the collective body of nutraceutical studies, the number of studies included in this explicit review after a systematic review of the medical literature was less than what would have been expected. Secondly, there are two aspects including possible publication bias and the selected search terms that could have influenced the results of the review. Some unpublished abstracts and articles have not been included due to their unavailability. Further, we nominated only the English and Persian language due to limited resources, which in turn would increase bias. These may significantly reduce the size of our sample and therefore our capacity to explain statistically important findings. Another weakness of this review was due to the heterogeneity of the selected studies which considered a variety of outcome events. The confounding effects of variations in background polyphenol intake among the studies must be considered in interpreting the results. As a final point, there may be some probable parts not considered in the current systematic review, such as the magnitude of IQ, region, diet, and race. Furthermore, this review relies on the findings of available published studies, so any limitations of these studies are unavoidable limitations of our review study as well. Despite the specified limitations, this systematic review has been the first systematic review of clinical trials investigating the effect of berry-based dietary interventions on cognitive function.

In included trials, the highest duration of berry-based food or supplement administration was 6 months. Furthermore, most studies had relatively low sample sizes. Future investigations should attempt to clarify the potential special effects of berry-based food or supplement consumption in a prolonged period of treatment to distinguish possible significant enhancements in cognition. Furthermore, the safety of these interventions should be described by means of a systematic method of recording probable adverse events based on a good clinical practice.

This review intended to collect and discuss scientific evidence concerning the beneficial role of consumption of berry-based products on the prevention of cognitive decline. In summary, from available human studies, intake of whole berries or berry-based products, principally consumed as fresh or frozen fruit, or as unsweetened beverages, may be endorsed as part of a healthy dietary approach to preventing cognitive decline among the elderly with healthy cognition or MCI. The future well-controlled dietary intervention-based investigations, preferably clinical trials, should more carefully choose appropriate supplements (as crude materials or whole berries) with different dosing regimens to determine the accurate dose. Individualized dietary and nutritional recommendations, definitely through metabotypes determination, seem to be an achievable approach for taking full advantage of any benefits provided by berries and their polyphenols. 
Received: 29 May 2021; Accepted: 8 February 2022

Published online: 25 February 2022

\section{References}

1. Kinsella, K. \& Velkoff, V. A. US Census Bureau, Series P95/01-1, an Aging World. (Government Printing Office, 2001).

2. http://www.who.int/mediacentre/factsheets/fs362/en/.

3. Thies, W. \& Bleiler, L. Vol. 9 208-245 (Alzheimers Dement 2013).

4. Hebert, L. E., Beckett, L. A., Scherr, P. A. \& Evans, D. A. Annual incidence of Alzheimer disease in the United States projected to the years 2000 through 2050. Alzheimer Dis. Assoc. Disord. 15, 169-173. https://doi.org/10.1097/00002093-200110000-00002 (2001).

5. Wang, Z., Pang, Y., Liu, J., Wang, J. \& Xie, Z. Association of healthy lifestyle with cognitive function among Chinese older adults. Eur. J. Clin. Nutr. 75, 325-334. https://doi.org/10.1038/s41430-020-00785-2 (2021).

6. Parrott, M. D. et al. The association between dietary pattern adherence, cognitive stimulating lifestyle, and cognitive function among older adults from the Quebec longitudinal study on nutrition and successful aging. J. Gerontol. Ser. B Psychol. Sci. Soc. Sci. 76, 444-450. https://doi.org/10.1093/geronb/gbaa178 (2021).

7. Jin, X. et al. Association of APOE $\varepsilon 4$ genotype and lifestyle with cognitive function among Chinese adults aged 80 years and older: A cross-sectional study. PLoS Med. 18, e1003597 (2021).

8. Appel, L. J. Dietary patterns and longevity: Expanding the blue zones. Circulation 118, 214-215 (2008).

9. Buettner, D. \& Skemp, S. Blue zones: Lessons from the world's longest lived. Am. J. Lifestyle Med. 10, 318-321 (2016).

10. Wu, L. \& Sun, D. Adherence to Mediterranean diet and risk of developing cognitive disorders: An updated systematic review and meta-analysis of prospective cohort studies. Sci. Rep. 7, 1-9 (2017).

11. Paleologos, M., Cumming, R. G. \& Lazarus, R. Cohort study of vitamin C intake and cognitive impairment. Am. J. Epidemiol. 148, 45-50 (1998).

12. Grodstein, F., Chen, J. \& Willett, W. C. High-dose antioxidant supplements and cognitive function in community-dwelling elderly women. Am. J. Clin. Nutr. 77, 975-984 (2003).

13. Wu, S. et al. Omega-3 fatty acids intake and risks of dementia and Alzheimer's disease: A meta-analysis. Neurosci. Biobehav. Rev. 48, 1-9 (2015).

14. Godos, J., Caraci, F., Castellano, S., Currenti, W. \& Galvano, F. Association Between Dietary Flavonoids Intake and Cognitive Function in an Italian. Cohort https://doi.org/10.3390/biom10091300 (2020).

15. Morris, M., Evans, D., Tangney, C., Bienias, J. \& Wilson, R. Associations of vegetable and fruit consumption with age-related cognitive change. Neurology 67, 1370-1376 (2006).

16. Feng, L. et al. Tea drinking and cognitive function in oldest-old Chinese. J. Nutr. Health Aging 16, 754-758. https://doi.org/10. 1007/s12603-012-0077-1 (2012).

17. Sugiyama, K. et al. Association between coffee consumption and incident risk of disabling dementia in elderly Japanese: The Ohsaki Cohort 2006 Study. J. Alzheimers Dis. 50, 491-500 (2016)

18. Wu, L. \& Sun, D. Meta-analysis of milk consumption and the risk of cognitive disorders. Nutrients 8, 824 (2016)

19. Sohal, R. S. \& Weindruch, R. Oxidative stress, caloric restriction, and aging. Science 273, 59-63 (1996).

20. Joseph, J. A. et al. Age-related neurodegeneration and oxidative stress: Putative nutritional intervention. Neurol. Clin. 16, 747-755 (1998).

21. Halliwell, B. Reactive oxygen species and the central nervous system. J. Neurochem. 59, 1609-1623 (1992).

22. Spencer, J. P. Food for thought: The role of dietary flavonoids in enhancing human memory, learning and neuro-cognitive performance: Symposium on 'Diet and mental health'. Proc. Nutr. Soc. 67, 238-252 (2008).

23. Joseph, J. A., Shukitt-Hale, B. \& Casadesus, G. Reversing the deleterious effects of aging on neuronal communication and behavior: Beneficial properties of fruit polyphenolic compounds. Am. J. Clin. Nutr. 81, 313S-316S (2005).

24. Spencer, J. P., Vauzour, D. \& Rendeiro, C. Flavonoids and cognition: The molecular mechanisms underlying their behavioural effects. Arch. Biochem. Biophys. 492, 1-9 (2009).

25. Spencer, J. P. Flavonoids: Modulators of brain function?. Br. J. Nutr. 99, ES60-ES77 (2008)

26. Spencer, J. P. Flavonoids and brain health: Multiple effects underpinned by common mechanisms. Genes Nutr. 4, 243-250 (2009).

27. Willis, L. M., Shukitt-Hale, B. \& Joseph, J. A. Recent advances in berry supplementation and age-related cognitive decline. Curr. Opin. Clin. Nutr. Metab. Care 12, 91-94 (2009).

28. Pandey, K. B. \& Rizvi, S. I. Plant polyphenols as dietary antioxidants in human health and disease. Oxid. Med. Cell. Longev. 2, 270-278 (2009).

29. Lau, F. C., Bielinski, D. F. \& Joseph, J. A. Inhibitory effects of blueberry extract on the production of inflammatory mediators in lipopolysaccharide-activated BV2 microglia. J. Neurosci. Res. 85, 1010-1017 (2007).

30. Brewer, G. J. et al. Age-related toxicity of amyloid-beta associated with increased pERK and pCREB in primary hippocampal neurons: Reversal by blueberry extract. J. Nutr. Biochem. 21, 991-998 (2010).

31. Skrovankova, S., Sumczynski, D., Mlcek, J., Jurikova, T. \& Sochor, J. Bioactive compounds and antioxidant activity in different types of berries. Int. J. Mol. Sci. 16, 24673-24706 (2015).

32. Calvano, A. et al. Dietary berries, insulin resistance and type 2 diabetes: An overview of human feeding trials. Food Funct. 10, 6227-6243. https://doi.org/10.1039/c9fo01426h (2019).

33. USDA Database for the Flavonoid content of selected foods, Release 3.1 Dec. 2013 with revisions May 2014, https://www.ars. usda.gov/ARSUserFiles/80400525/Data/Flav/Flav_R03-1.pdf.

34. Li, P. et al. Protective effects of anthocyanins on neurodegenerative diseases. Trends Food Sci. Technol. 117, 205-217 (2021).

35. Kay, C. D., Pereira-Caro, G., Ludwig, I. A., Clifford, M. N. \& Crozier, A. Anthocyanins and flavanones are more bioavailable than previously perceived: A review of recent evidence. Ann. Rev. Food Sci. Technol. 8, 155-180 (2017).

36. Joseph, J. A., Shukitt-Hale, B. \& Lau, F. C. Fruit polyphenols and their effects on neuronal signaling and behavior in senescence. Ann. N. Y. Acad. Sci. 1100, 470-485 (2007).

37. Kay, C. D. \& Holub, B. J. The effect of wild blueberry (Vaccinium angustifolium) consumption on postprandial serum antioxidant status in human subjects. Br. J. Nutr. 88, 389-397 (2002).

38. Rodriguez-Mateos, A. et al. Intake and time dependence of blueberry flavonoid-induced improvements in vascular function: A randomized, controlled, double-blind, crossover intervention study with mechanistic insights into biological activity. Am. J. Clin. Nutr. 98, 1179-1191 (2013).

39. Bell, L., Lamport, D. J., Butler, L. T. \& Williams, C. M. A study of glycaemic effects following acute anthocyanin-rich blueberry supplementation in healthy young adults. Food Funct. 8, 3104-3110 (2017)

40. Miller, M. G. \& Shukitt-Hale, B. Berry fruit enhances beneficial signaling in the brain. J. Agric. Food Chem. 60, 5709-5715 (2012).

41. Williamson, G. \& Clifford, M. N. Colonic metabolites of berry polyphenols: The missing link to biological activity?. Br. J. Nutr. 104, S48-S66 (2010).

42. Shukitt-Hale, B. et al. Blueberry polyphenols attenuate kainic acid-induced decrements in cognition and alter inflammatory gene expression in rat hippocampus. Nutr. Neurosci. 11, 172-182 (2008). 
43. Joseph, J. A. et al. Differential protection among fractionated blueberry polyphenolic families against DA-, A $\beta 42$-and LPSInduced decrements in Ca2+ buffering in primary hippocampal cells. J. Agric. Food Chem. 58, 8196-8204 (2010).

44. Thangthaeng, N., Poulose, S. M., Miller, M. G. \& Shukitt-Hale, B. Preserving brain function in aging: The anti-glycative potential of berry fruit. NeuroMol. Med. 18, 465-473 (2016).

45. Goyarzu, P. et al. Blueberry supplemented diet: Effects on object recognition memory and nuclear factor-kappa B levels in aged rats. Nutr. Neurosci. 7, 75-83 (2004).

46. Joseph, J. A. et al. Reversals of age-related declines in neuronal signal transduction, cognitive, and motor behavioral deficits with blueberry, spinach, or strawberry dietary supplementation. J. Neurosci. 19, 8114-8121 (1999).

47. Shukitt-Hale, B. et al. In Society for Neuroscience Abstract.

48. Shukitt-Hale, B., Smith, D. E., Meydani, M. \& Joseph, J. A. The effects of dietary antioxidants on psychomotor performance in aged mice. Exp. Gerontol. 34, 797-808 (1999).

49. Joseph, J. A. et al. Long-term dietary strawberry, spinach, or vitamin E supplementation retards the onset of age-related neuronal signal-transduction and cognitive behavioral deficits. J. Neurosci. 18, 8047-8055 (1998).

50. Pedersen, C. B. et al. Effects of blueberry and cranberry juice consumption on the plasma antioxidant capacity of healthy female volunteers. Eur. J. Clin. Nutr. 54, 405-408 (2000).

51. Tulipani, S., Mezzetti, B. \& Battino, M. Impact of strawberries on human health: Insight into marginally discussed bioactive compounds for the Mediterranean diet. Public Health Nutr. 12, 1656-1662 (2009).

52. Ellis, C. L., Edirisinghe, I., Kappagoda, T. \& Burton-Freeman, B. Attenuation of meal-induced inflammatory and thrombotic responses in overweight men and women after 6-week daily strawberry (Fragaria) intake: A randomized placebo-controlled trial. J. Atheroscler. Thromb. 18, 318-327 (2011).

53. Krikorian, R. et al. Blueberry supplementation improves memory in older adults. J. Agric. Food Chem. 58, 3996-4000 (2010).

54. Higgins, J. P. \& Green, S. Cochrane Handbook for Systematic Reviews of Interventions Vol. 4 (Wiley, New York, 2011).

55. Moher, D., Liberati, A., Tetzlaff, J. \& Altman, D. G. Preferred reporting items for systematic reviews and meta-analyses: The PRISMA statement. Ann. Intern. Med. 151, 264-269, W264 (2009).

56. Phan, K., Tian, D. H., Cao, C., Black, D. \& Yan, T. D. Systematic review and meta-analysis: Techniques and a guide for the academic surgeon. Ann. Cardiothorac. Surg. 4, 112-122. https://doi.org/10.3978/j.issn.2225-319X.2015.02.04 (2015).

57. Sackett, D. L. In Seminars in Perinatology. 3-5 (Elsevier).

58. Higgins, J. P. et al. The Cochrane collaboration's tool for assessing risk of bias in randomised trials. BMJ 343, d5928 (2011).

59. Dodd, G. F., Williams, C. M., Butler, L. T. \& Spencer, J. P. Acute effects of flavonoid-rich blueberry on cognitive and vascular function in healthy older adults. Nutr. Healthy Aging 5, 119-132 (2019).

60. Harvey, P. D. \& Mohs, R. C. In Functional neurobiology of aging 53-63 (University of Miami, 2001).

61. Krikorian, R. et al. Cognitive performance in relation to urinary anthocyanins and their flavonoid-based products following blueberry supplementation in older adults at risk for dementia. J. Funct. Foods 64, 1-10 (2020).

62. Schrager, M. A., Hilton, J., Gould, R. \& Kelly, V. E. Effects of blueberry supplementation on measures of functional mobility in older adults. Appl. Physiol. Nutr. Metab. 40, 543-549 (2015).

63. Bensalem, J. et al. Polyphenols from grape and blueberry improve episodic memory in healthy elderly with lower level of memory performance: A bicentric double-blind, randomized, placebo-controlled clinical study. J. Gerontol. A Biol. Sci. Med. Sci. 74, 996-1007. https://doi.org/10.1093/gerona/gly166 (2019).

64. Bowtell, J. L., Aboo-Bakkar, Z., Conway, M. E., Adlam, A. R. \& Fulford, J. Enhanced task-related brain activation and resting perfusion in healthy older adults after chronic blueberry supplementation. Appl. Physiol. Nutr. Metab. 42, 773-779. https://doi. org/10.1139/apnm-2016-0550 (2017).

65. Whyte, A., Cheng, N., Fromentin, E. \& Williams, C. A randomized, double-blinded, placebo-controlled study to compare the safety and efficacy of low dose enhanced wild blueberry powder and wild blueberry extract (ThinkBlue ${ }^{\mathrm{et}}$ ) in maintenance of episodic and working memory in older adults. Nutrients 10, 660 (2018).

66. Miller, M. G., Hamilton, D. A., Joseph, J. A. \& Shukitt-Hale, B. Dietary blueberry improves cognition among older adults in a randomized, double-blind, placebo-controlled trial. Eur. J. Nutr. 57, 1169-1180. https://doi.org/10.1007/s00394-017-1400-8 (2018).

67. Delis, D., Kramer, J., Kaplan, E. \& Ober, B. California Verbal Learning Test (The Psychological Corporation, San Antonio, 2000).

68. McNamara, R. K. et al. Cognitive response to fish oil, blueberry, and combined supplementation in older adults with subjective cognitive impairment. Neurobiol. Aging 64, 147-156 (2018).

69. Cowan, N. Working memory underpins cognitive development, learning, and education. Educ. Psychol. Rev. 26, 197-223 (2014).

70. Nilsson, A., Salo, I., Plaza, M. \& Bjorck, I. Effects of a mixed berry beverage on cognitive functions and cardiometabolic risk markers; A randomized cross-over study in healthy older adults. PLOS ONE 12, e0188173. https://doi.org/10.1371/journal.pone. 0188173 (2017).

71. Radeborg, K., Briem, V. \& Hedman, L. R. The effect of concurrent task difficulty on working memory during simulated driving. Ergonomics 42, 767-777 (1999).

72. Bell, L., Lamport, D. J., Field, D. T., Butler, L. T. \& Williams, C. M. Practice effects in nutrition intervention studies with repeated cognitive testing. Nutr. Healthy Aging 4, 309-322 (2018).

73. Whyte, A. R., Schafer, G. \& Williams, C. M. The effect of cognitive demand on performance of an executive function task following wild blueberry supplementation in 7 to 10 years old children. Food Funct. 8, 4129-4138 (2017).

74. Visser, M., Jefferies, E., Embleton, K. V. \& Lambon Ralph, M. A. Both the middle temporal gyrus and the ventral anterior temporal area are crucial for multimodal semantic processing: Distortion-corrected fMRI evidence for a double gradient of information convergence in the temporal lobes. J. Cogn. Neurosci. 24, 1766-1778. https://doi.org/10.1162/jocn_a_00244 (2012).

75. Li, J. et al. The regional neuronal activity in left posterior middle temporal gyrus is correlated with the severity of chronic aphasia. Neuropsychiatr. Dis. Treat. 13, 1937-1945. https://doi.org/10.2147/ndt.s140091 (2017).

76. Goel, V., Gold, B., Kapur, S. \& Houle, S. Neuroanatomical correlates of human reasoning. J. Cogn. Neurosci. 10, 293-302. https:// doi.org/10.1162/089892998562744 (1998).

77. Xu, J. et al. Tractography-based parcellation of the human middle temporal gyrus. Sci. Rep. 5, 18883. https://doi.org/10.1038/ srep18883 (2015).

78. Stoeckel, C., Gough, P. M., Watkins, K. E. \& Devlin, J. T. Supramarginal gyrus involvement in visual word recognition. Cortex J. Devot. Study Nerv. Syst. Behav. 45, 1091-1096. https://doi.org/10.1016/j.cortex.2008.12.004 (2009).

79. Wada, S. \& Honma, M. Volume of the right supramarginal gyrus is associated with a maintenance of emotion recognition ability. 16, e0254623. https://doi.org/10.1371/journal.pone.0254623 (2021).

80. Schaal, N. K., Pollok, B. \& Banissy, M. J. Hemispheric differences between left and right supramarginal gyrus for pitch and rhythm memory. Sci. Rep. 7, 42456. https://doi.org/10.1038/srep42456 (2017).

81. Nieuwenhuys, R., Voogd, J. \& Van Huijzen, C. The Human Central Nervous System: A Synopsis and Atlas (Springer, New York, 2007).

82. Fadiga, L., Craighero, L. \& Roy, A. In Broca’s Area: A Speech Area? (eds Grodzinsky, E. Y. \& Amunts, K.) 137-152 (Oxford University press New York, NY, 2006).

83. Wakita, M. Broca's area processes the hierarchical organization of observed action. Front. Hum. Neurosci. 7, 937 (2014).

84. Papez, J. W. A proposed mechanism of emotion. Arch. Neurol. Psychiatry 38, 725-743 (1937). 
85. Kiehl, K. A., Liddle, P. F. \& Hopfinger, J. B. Error processing and the rostral anterior cingulate: an event-related fMRI study. Psychophysiology 37, 216-223 (2000).

86. Bush, G., Luu, P. \& Posner, M. I. Cognitive and emotional influences in anterior cingulate cortex. Trends Cogn. Sci. 4, 215-222 (2000).

87. MacDonald, A. W., Cohen, J. D., Stenger, V. A. \& Carter, C. S. Dissociating the role of the dorsolateral prefrontal and anterior cingulate cortex in cognitive control. Science 288, 1835-1838 (2000).

88. Mulert, C., Gallinat, J., Dorn, H., Herrmann, W. M. \& Winterer, G. The relationship between reaction time, error rate and anterior cingulate cortex activity. Int. J. Psychophysiol. 47, 175-183 (2003).

89. Naito, E. et al. Fast reaction to different sensory modalities activates common fields in the motor areas, but the anterior cingulate cortex is involved in the speed of reaction. J. Neurophysiol. 83, 1701-1709 (2000).

90. Uddin, L. Q., Nomi, J. S., Hébert-Seropian, B., Ghaziri, J. \& Boucher, O. Structure and function of the human Insula. J. Clin. Neurophysiol. 34, 300-306. https://doi.org/10.1097/wnp.0000000000000377 (2017).

91. Fama, R. \& Sullivan, E. V. Thalamic structures and associated cognitive functions: Relations with age and aging. Neurosci. Biobehav. Rev. 54, 29-37. https://doi.org/10.1016/j.neubiorev.2015.03.008 (2015).

92. Boespflug, E. L. et al. Enhanced neural activation with blueberry supplementation in mild cognitive impairment. Nutr. Neurosci. 21, 297-305. https://doi.org/10.1080/1028415X.2017.1287833 (2018).

93. Padmala, S. \& Pessoa, L. Interactions between cognition and motivation during response inhibition. Neuropsychologia 48, 558-565 (2010).

94. Wen, J. et al. Evaluating the roles of left middle frontal gyrus in word production using electrocorticography. Neurocase 23, 263-269 (2017).

95. Koyama, M. S., O'Connor, D., Shehzad, Z. \& Milham, M. P. Differential contributions of the middle frontal gyrus functional connectivity to literacy and numeracy. Sci. Rep. 7, 1-13 (2017).

96. Andersson, M., Ystad, M., Lundervold, A. \& Lundervold, A. J. Correlations between measures of executive attention and cortical thickness of left posterior middle frontal gyrus-a dichotic listening study. Behav. Brain Funct. 5, 1-9 (2009).

97. Bzdok, D. et al. Left inferior parietal lobe engagement in social cognition and language. Neurosci. Biobehav. Rev. 68, 319-334 (2016).

98. Binder, J. R., Desai, R. H., Graves, W. W. \& Conant, L. L. Where is the semantic system? A critical review and meta-analysis of 120 functional neuroimaging studies. Cerebral Cortex (New York, N.Y. : 1991) 19, 2767-2796. https://doi.org/10.1093/cercor/ bhp055 (2009).

99. Zheng, J. et al. Effects and mechanisms of fruit and vegetable juices on cardiovascular diseases. Int. J. Mol Sci. https://doi.org/ 10.3390/ijms18030555 (2017).

100. Ruxton, C. Smoothies: One portion or two?. Nutr. Bull. 33, 129-132 (2008).

101. Kamiloglu, S. et al. Home processing of tomatoes (Solanum lycopersicum): Effects on in vitro bioaccessibility of total lycopene, phenolics, flavonoids, and antioxidant capacity. J. Sci. Food Agric. 94, 2225-2233. https://doi.org/10.1002/jsfa.6546 (2014).

102. Porrini, M., Riso, P. \& Testolin, G. Absorption of lycopene from single or daily portions of raw and processed tomato. Br. J. Nutr. 80, 353-361. https://doi.org/10.1079/096582198388300 (1998).

103. Nile, S. H. \& Park, S. W. Edible berries: Bioactive components and their effect on human health. Nutrition 30, 134-144 (2014).

104. Szajdek, A. \& Borowska, E. Bioactive compounds and health-promoting properties of berry fruits: A review. Plant Foods Hum. Nutr. 63, 147-156 (2008).

105. Basu, A. Role of berry bioactive compounds on lipids and lipoproteins in diabetes and metabolic syndrome. Nutrients https:// doi.org/10.3390/nu11091983 (2019).

106. Bartosz, G. \& Sadowska-Bartosz, I. In Studies on Psychiatric Disorders 1-39 (Springer, 2015).

107. Miyazaki, K., Makino, K., Iwadate, E., Deguchi, Y. \& Ishikawa, F. Anthocyanins from purple sweet potato Ipomoea batatas cultivar Ayamurasaki suppress the development of atherosclerotic lesions and both enhancements of oxidative stress and soluble vascular cell adhesion molecule-1 in apolipoprotein E-deficient mice. J. Agric. Food Chem. 56, 11485-11492 (2008).

108. Wang, H., Cao, G. \& Prior, R. L. Oxygen radical absorbing capacity of anthocyanins. J. Agric. Food Chem. 45, 304-309 (1997).

109. Li, H. et al. Highly pigmented vegetables: Anthocyanin compositions and their role in antioxidant activities. Food Res. Int. 46, 250-259 (2012).

110. Geng, J. et al. Blood-brain barrier disruption induced cognitive impairment is associated with increase of inflammatory cytokine. Front. Aging Neurosci. 10, 129 (2018).

111. Baker, R. G., Hayden, M. S. \& Ghosh, S. NF-кB, inflammation, and metabolic disease. Cell Metab. 13, 11-22 (2011).

112. Pomari, E., Stefanon, B. \& Colitti, M. Effect of plant extracts on $\mathrm{H}_{2} \mathrm{O}_{2}$-induced inflammatory gene expression in macrophages. J. Inflamm. Res. 7, 103 (2014).

113. Lietti, A., Cristoni, A. \& Picci, M. Studies on Vaccinium myrtillus anthocyanosides. I. Vasoprotective and antiinflammatory activity. Arzneimittel-Forschung 26, 829-832 (1976).

114. Guo, H. \& Ling, W. The update of anthocyanins on obesity and type 2 diabetes: Experimental evidence and clinical perspectives. Rev. Endocr. Metab. Disord. 16, 1-13 (2015).

115. Denis, M.-C. et al. Prevention of oxidative stress, inflammation and mitochondrial dysfunction in the intestine by different cranberry phenolic fractions. Clin. Sci. 128, 197-212 (2015).

116. di Gesso, J. L. et al. Flavonoid metabolites reduce tumor necrosis factor- $\alpha$ secretion to a greater extent than their precursor compounds in human THP-1 monocytes. Mol. Nutr. Food Res. 59, 1143-1154 (2015).

117. Hassimotto, N. M. A. et al. Inhibition of carrageenan-induced acute inflammation in mice by oral administration of anthocyanin mixture from wild mulberry and cyanidin-3-glucoside. BioMed Res. Int. 2013, 1-10 (2013).

118. Warner, E. F. et al. Signatures of anthocyanin metabolites identified in humans inhibit biomarkers of vascular inflammation in human endothelial cells. Mol. Nutr. Food Res. https://doi.org/10.1002/mnfr.201700053 (2017).

119. Del Rio, D. et al. Dietary (poly) phenolics in human health: Structures, bioavailability, and evidence of protective effects against chronic diseases. Antioxid. Redox Signal. 18, 1818-1892 (2013).

120. Cardona, F., Andrés-Lacueva, C., Tulipani, S., Tinahones, F. J. \& Queipo-Ortuño, M. I. Benefits of polyphenols on gut microbiota and implications in human health. J. Nutr. Biochem. 24, 1415-1422 (2013).

121. Duda-Chodak, A., Tarko, T., Satora, P. \& Sroka, P. Interaction of dietary compounds, especially polyphenols, with the intestinal microbiota: A review. Eur. J. Nutr. 54, 325-341 (2015).

122. Vanamala, J. K., Knight, R. \& Spector, T. D. Can your microbiome tell you what to eat?. Cell Metab. 22, 960-961 (2015).

123. Westfall, S. et al. Microbiome, probiotics and neurodegenerative diseases: Deciphering the gut brain axis. Cell. Mol. Life Sci. 74, 3769-3787 (2017)

\section{Acknowledgements}

The authors would like to appreciate Dr. Azizeh Farshbaf Khalili, Dr. Hanieh Salehi Pourmehr and Mrs. Fahimeh Bakhtiari for her assistance with this study. The research protocol was approved and supported by Student Research Committee, Tabriz University of Medical Sciences (grant number: 62557). 


\section{Author contributions}

N.B., N.D., Y.S., and M.H.contributed to the conception and design of the study, data analysis and interpretation, and drafting of the manuscript. N.B. collected all data and contributed to data interpretation and drafting the manuscript. N.B. and N.D. drafted the first manuscript. N.B., N.D. and M.H.contributed in final drafting of manuscript. All authors read and approved the final manuscript.

\section{Funding}

The research protocol was approved \& supported by the Student Research Committee, Tabriz University of Medical Sciences (registration code: 62557). The study sponsor was not included in the study design; data collection, analysis, and interpretation; the writing of the manuscript; nor in the decision to submit the manuscript for publication.

\section{Competing interests}

The authors declare no competing interests.

\section{Additional information}

Correspondence and requests for materials should be addressed to N.D.

Reprints and permissions information is available at www.nature.com/reprints.

Publisher's note Springer Nature remains neutral with regard to jurisdictional claims in published maps and institutional affiliations.

(c) (i) Open Access This article is licensed under a Creative Commons Attribution 4.0 International License, which permits use, sharing, adaptation, distribution and reproduction in any medium or format, as long as you give appropriate credit to the original author(s) and the source, provide a link to the Creative Commons licence, and indicate if changes were made. The images or other third party material in this article are included in the article's Creative Commons licence, unless indicated otherwise in a credit line to the material. If material is not included in the article's Creative Commons licence and your intended use is not permitted by statutory regulation or exceeds the permitted use, you will need to obtain permission directly from the copyright holder. To view a copy of this licence, visit http://creativecommons.org/licenses/by/4.0/.

(c) The Author(s) 2022 